\title{
Distribution of Carbohydrate Epitopes among Disjoint Subsets of Leech Sensory Afferent Neurons
}

\author{
Karl Zipser, ${ }^{1}$ Michelle Erhardt, ${ }^{2}$ Jianxiong Song, ${ }^{2}$ Robert N. Cole, ${ }^{3}$ and Birgit Zipser ${ }^{2}$ \\ 'Department of Brain and Cognitive Sciences, Massachusetts Institute of Technology, Cambridge, Massachusetts 02139, \\ 2Department of Physiology, Michigan State University, East Lansing, Michigan 48824, and ${ }^{3}$ Department of Biochemistry \\ and Molecular Genetics, University of Alabama at Birmingham, Birmingham, Alabama 35294
}

\begin{abstract}
Carbohydrate recognition plays an important role in the development of normal projections of sensory afferent neurons in the leech CNS. Four different carbohydrate epitopes are expressed by sensory afferents on their $130 \mathrm{kDa}$ surface proteins: all sensory afferents share a common carbohydrate epitope (CE0) that helps them to enter and project diffusely across the synaptic neuropil; a restricted expression of three other carbohydrate epitopes (CE1, CE2, and CE3) serves to distinguish three subsets of sensory afferents. We examined the subsets of sensory afferents defined by their subset carbohydrate epitopes in the leech lip, skin, gut, and CNS. We established that the CE1, CE2, and CE3 subset epitopes define disjoint subsets of neurons by double labeling sensory afferents with monoclonal antibodies for different pairs of subset epitopes. We found that CE2 and CE3 afferents populate the lip and skin, but not the gut, and that these two subsets of sensory afferents have convergent projection patterns in the CNS. We found that CE1 afferents populate the gut and skin, but not lips; furthermore, their CNS projections diverge from those of CE2 and CE3 afferents. Our data fit the hypothesis that these carbohydrate epitopes are related to sensory modality of afferent subsets.

IKey words: leech, development, carbohydrates, lectin, synapse formation, process outgrowth, surface glycoprotein]
\end{abstract}

Because groups or subsets of neurons can be distinguished by their different surface oligosaccharides (as reviewed in Jessell et al., 1990; Scott et al., 1990; Key and Akeson, 1991; Schwarting et al., 1992; Whitlock, 1993), diversity in carbohydrate structure may help neurons to recognize each other as they form functional networks. For example, sensory afferents projecting to different regions in the rat dorsal horn express different oligosaccharides (Jessell and Dodd, 1985). Likewise, in the leech, sensory afferent neurons associated with epithelial layers display different carbohydrates identifiable by monoclonal antibodies on their cell surface. One of these carbohydrates, a mannosecontaining epitope (carbohydrate epitope 0, CEO), appears to label all these neurons (Peinado et al., 1990; present report),

\footnotetext{
Received Oct. 18, 1993; revised Jan. 19, 1994; accepted Jan. 27, 1994.

We thank C. Osborn and M. Rheuben for helpful comments on the manuscript. This work was supported by a National Institute of Health Grant NS25117 and an ONR fellowship to K.Z.

Correspondence should be addressed to Birgit Zipser, Department of Physiology, Giltner Hall, Michigan State University, East Lansing, MI 48824-1101.

Copyright (C) 1994 Society for Neuroscience $0270-6474 / 94 / 144481-13 \$ 05.00 / 0$
}

and therefore we consider it to be a marker for the full set of the sensory afferents studied here. In contrast, three other carbohydrate epitopes (CEI, CE2, and CE3) are expressed only by subsets of afferents and are therefore termed "subset epitopes" (Peinado et al., 1987; Bajt et al., 1990; evidence for the carbohydrate character of CE3 is presented in this report).

Anatomical studies of the projections of leech sensory afferents in the synaptic regions of the CNS showed that the distribution of various carbohydrate epitopes, in some cases, delineated separate afferent projection patterns (Peinado et al., 1987, 1990), suggesting that carbohydrate epitopes play some role in the establishment of these patterns. Developmental studies with the leech embryo have since confirmed this hypothesis. For example, an early step in the formation of sensory afferent patterns, the defasciculation and dispersal of afferents across the synaptic neuropil, is mediated by a mannose-specific recognition involving their shared marker, CE0 (Zipser et al., 1989; Zipser and Cole, 1991). In another example, the segregation of an afferent subset, for example, the CE1 subset to a restricted neuropil region, is mediated by its subset marker, CEl (Song and Zipser, 1993). Interestingly, while sensory afferents express these different carbohydrate epitopes over their entire surface, delicately manipulating the molecular interactions of these epitopes only perturbs afferent growth in the synaptic neuropil, but not in axonal tracts leading into or out of the neuropil. This suggests these carbohydrate epitopes participate in the recognition process by which sensory afferent neurons select their synaptic contacts.

Although it has been established that carbohydrate markers distinguish subsets of neurons in a way relevant for development of neural patterns, little is known about how neurons that constitute the different subsets differ from each other. Full understanding of the significance of carbohydrate markers in neural development requires that we understand the properties of sensory afferents that they delineate. The purpose of this report is to characterize afferents of three major carbohydrate-defined subsets by examining their distribution, both in peripheral tissues and within the CNS. Our analysis suggests that the subset carbohydrate epitopes could code sensory modality of afferent subsets.

\section{Materials and Methods}

Experiments were performed on two leech species, Hirudo medicinalis and Haemopis marmorata, which are bred in our laboratory (Cole et al., 1989; Zipser and Cole, 1991). For biochemical experiments we used the nerve cords (CNS) of adult Haemopis marmorata. Immunocytochemical experiments were performed with Hirudo medicinalis: body 
walls were dissected from embryos close to hatching ( $23 \mathrm{~d}$ ), and ganglia from nerve cords of juvenile and adult leeches.

Antibodies. The monoclonal antibodies (mAbs) used for this study were generated by our laboratory against the leech species Haemopis marmorata (Zipser and McKay, 1981; Flaster et al., 1983; Hogg et al., 1983). The antibodies recognize similar $130 \mathrm{kDa}$ surface glycoproteins on sensory afferent neurons in Haemopis marmorata and Hirudo medicinalis (McGlade-McCulloh et al., 1990). Four mAbs were used to study the carbohydrate epitopes (CE) on the surface of sensory afferent neurons: $m A b s \operatorname{Lan} 3-2, \operatorname{Laz} 2-369, \operatorname{Laz} 7-79$, and $\operatorname{Lan} 2-3$ bound to carbohydrate epitopes $\mathrm{CEO}, \mathrm{CE} 1, \mathrm{CE} 2$, and CE3, respectively. mAb Laz1-1 binds to an internal antigen of sensory afferents (Moore et al., 1988). For staining, Lan 3-2 was used as ascites fluid $(7 \mathrm{mg} / \mathrm{ml})$, diluted 1:4000; the other $\mathrm{mAbs}$ were used as tissue culture supernatant $(20 \mu \mathrm{g} / \mathrm{ml})$, diluted 1:10 (except in sugar blocking studies; see below). To serve in double-labeling experiments of fixed adult ganglia, mAbs were purified from ascites fluid and biotinylated by following methods described by Harlow and Lane (1988). To serve in double-labeling experiments of live juvenile nerve cords, mAbs were directly labeled with 5-carboxyfluorescein and 5-carboxytetramethylrhodamine (Molecular Probes, OR) following methods described by Harlow and Lane (1988).

Immunocytochernistry on live and fixed tissue. Embryonic skin, because it is more permeable to antibody penetration than adult skin, was used to study the cell bodies of sensory afferents. Prior to dissection, the cuticle was loosened from the skin by incubating embryos in papain (see below). Then, the embryos were opened at the dorsal midline, the nerve cord and other organs were removed, and the cuticle was pulled off. The projections of sensory afferents were studied in midbody ganglia of nerve cords of juvenile and adult leeches. Nerve cords were dissected from alcohol-anesthetized leeches and pinned out on Sylgard. The capsule over each ganglion was removed with forceps. Embryonic skin and adult nerve cords were fixed in $4 \%$ paraformaldehyde in $0.1 \mathrm{~m}$ phosphate buffer, $\mathrm{pH} 7.4$, for $20 \mathrm{~min}$. Juvenile nerve cords were maintained in Leibovitz 15 (GIBCO Laboratories, NY) and stained live.

To permeabilize skin and adult ganglia, these tissues were treated prior to fixation with papain in PBS buffer (pH 7.4) containing $5 \mathrm{~mm}$ EGTA and fresh cysteine $(1.6 \mathrm{mg} / 10 \mathrm{ml})$. To loosen the cuticle from the skin, the whole embryo was treated with $6 \mu \mathrm{l}$ papain, $100 \mu \mathrm{l}$ buffer for $2-3 \mathrm{hr}$; adult ganglia were treated with $2 \mu \mathrm{l}$ papain, $100 \mu \mathrm{l}$ buffer for $15 \mathrm{~min}$. Mercuripapain (Worthington Biochemical Corp., NJ), used here as a source of papain, was preincubated in buffer for $0.5 \mathrm{hr}$ prior to digestion, so that the papain would be activated by the removal of mercury through EGTA. After the enzymatic digestion, the tissues were fixed and then further permeabilized with alcohol and xylene extraction.

All antibody incubation and washing steps involving fixed tissue were carried out in the presence of $2 \%$ Triton X-100 on a shaker at room temperature. Nonspecific binding was blocked with fetal calf serum present in the monoclonal antibody supernatant. However, ascites fluid, secondary antibodies, and avidin were routinely diluted into PBS containing 3\% bovine serum albumin (BSA), because fetal calf serum interferes with the avidin biotin interaction. MAbs were applied overnight, secondary antibodies for $2 \mathrm{hr}$, and avidin for $1 \mathrm{hr}$. When labeling neurons with just one $\mathrm{mAb}$, the $\mathrm{mAb}$ was visualized with biotinylated $\mathrm{F}\left(\mathrm{ab}^{\prime}\right)_{2}$ (1:100; Dako Corp., CA), followed by avidin-HRP (1:150; Vector Laboratories, CA) or avidin Texas red (1:1000). For double labeling neurons with two different $\mathrm{mAbs}$, the first $\mathrm{mAb}$ was visualized with RITC-conjugated anti-mouse IgG (1:50; Dako). The anti-mouse IgG was then blocked through an incubation with mouse IgG $(5 \%)$ for 0.5 $\mathrm{hr}$. Next, the second biotinylated $\mathrm{mAb}$ was applied for $4 \mathrm{hr}$, followed by avidin FITC (1:600). To double label live sensory afferent axons, juvenile nerve cords were maintained for $9 \mathrm{hr}$ in Leibovitz 15 in the presence of two different monoclonal antibodies, one directly conjugated to fluorescein and the other to rhodamine, Antibodies were washed out with a brief rinse, and the tissue was embedded live. Fluorescently stained tissue was embedded in $70 \%$ glycerol, $30 \%$ glycine buffer ( $\mathrm{pH}$ 9) to which saturating levels of $p$-phenylenediamine (Sigma, St. Louis, $\mathrm{MO}$ ) had been added to prevent the bleaching of fluorescence. Tissue incubated with avidin-HRP was treated with $0.03 \%$ diaminobenzidine $(\mathrm{DAB})$ and $\mathrm{H}_{2} \mathrm{O}_{2}$ until the desired intensity of the reaction product was achieved. DAB-stained tissue was embedded in Permount (Fisher Scientific Co., Fair Lawn, NJ) as whole-mounts or as sections. For sectioning, tissue was embedded in JB-4 (Polysciences, Inc., PA), following the manufacturers instructions, sectioned at $4 \mu \mathrm{m}$ with glass knives, collected on polylysine-coated slides in a drop of ethanol, dehydrated, and mounted.
Analysis of immunocytochemically stained tissue. The fluorescently stained tissue was analyzed on a Zeiss 10 Laser Scanning Confocal microscope or on the Odyssey Laser Confocal microscope (Noran Instruments, Madison, WI), using $40 \times, 60 \times$, or $100 \times$ oil-immersion objectives. The excitation and primary barrier filters for fluorescein were 488 and $515 \mathrm{~nm}$, respectively. The excitation and primary barrier filters for rhodamine were 529 and $550 \mathrm{~nm}$, respectively. In some instances, two additional bandpass filters (590 and $520-560 \mathrm{~nm}$; Noran Instruments) were used to control for bleed-through between fluorescein and rhodamine optics. The intensity of staining was analyzed using IMAGE-1 software (see below). The DAB-stained tissue was analyzed using a $100 \mathrm{x}$ Zeiss oil-immersion lens and bright-field optics on a Nikon optiphot. The number of cell bodies in the embryonic sensilla ( $23 \mathrm{~d}$ ) were counted on micrographs taken at $\mathbf{1 0}$ different focal planes through the width of a sensillum. We ignored round peripheral structures ( $20 \mu \mathrm{m}$ in diameter), projecting a thick duct ( $5 \mu \mathrm{m}$ wide), that are weakly cross-reactive with Lan 3-2 (Johansen et al., 1992), Laz2-369, Laz7-79, and Lan2-3 because we consider them to be gland cells. The relative areas of axonal tracts were estimated by excising tract regions from photomicrographs and weighing these with a Mettler balance.

Blocking of antibody binding with monosaccharides. Supernatant with mAb Lan2-3 was dilutcd 1:50 in PBS, 3\% BSA, 2\% Triton X-100, as this concentration was determined to give just below maximal antibody binding to carbohydrate epitope CE3. Galactose or $\beta$-methyl galactose were added at a concentration of $0.5 \mathrm{M}$ and this mixture was incubated for $3 \mathrm{hr}$ at room temperature.

For the histological assay, nerve cords were treated with Lan2-3 in the absence and presence of monosaccharides for $3 \mathrm{hr}$, washed, treated with biotinylated $F\left(a^{\prime}\right)_{2}(2 \mathrm{hr})$ and avidin Texas red $(1 \mathrm{hr})$, and then washed again and embedded. For each data point, 12 serial optical sections were taken from the connectives of a nerve cord. The sections were summed and measured for their intensity of antibody staining using IMAGE-1 (Universal Imaging Corp., West Chester, PA) and an EXCEL spreadsheet (Macintosh). Background was determined on sections of connectives that were incubated in the absence of Lan 2-3, but in the presence of sccondary antibody and Texas red. For biochemical assays, dot blots were prepared by blotting onto nitrocellulose $5 \mu \mathrm{g}$ of leech nerve cord homogenates and blocking the dots with $10 \%(\mathrm{v} / \mathrm{v})$ nonfat milk powder in PBS containing $0.05 \%$ Tween 20 . The antibody-sugar solution was applied to dot blots for $2 \mathrm{hr}$, rinsed and blocked in the milk blocking solution, and, finally, incubated for $1 \mathrm{hr}$ with ${ }^{125} \mathrm{I}$-labeled goat anti-mouse IgG $(300,000 \mathrm{cpm} / \mathrm{ml}$; ICN Biomedicals, Inc., CA). To quantify Lan 2-3 binding, dot blots were rinsed of unbound antibody and counted for bound reactivity in a gamma counter. Three replicas of four dot blots were used to determine Lan 2-3 binding in each sugar concentration. Background was determined on dot blots that were not incubated with $\mathrm{mAb}$ Lan 2-3 or on dots blots that were not blotted with leech nerve cord protein.

Lectin affinity chromatography. Fifteen nerve cords were solubilized in Tris-buffered salinc ( $\mathrm{pH}$ ) containing $0.5 \%$ Triton X-100, $1 \mathrm{~mm}$ $\mathrm{CaCl}_{2}, 1 \mathrm{mM} \mathrm{MgCl}, 1 \mathrm{mM} \mathrm{MnCl}$, and PMSF by a $1 \mathrm{hr}$ incubation on ice while shaking. The Triton X-100 extracts were applied to a $0.75 \mathrm{ml}$ column of lentil lectin (Sigma, St. Louis, MO). Columns were washed with $30 \mathrm{ml}$ buffer, the first $5 \mathrm{ml}$ of which were collected as void fraction. Columns were then eluted with $10 \mathrm{ml}$ of $0.5 \mathrm{M} \alpha$-methyl mannoside (Sigma, St. Louis, MO). Then, $2.5 \mathrm{ml}$ of eluant was collected as binding fractions. Protein from the fractions was alcohol precipitated and boiled in SDS-PAGE sample buffer (Laemmli, 1970). Proteins were then separated by electrophoresis on $7.5 \%$ SDS-PAGE and transferred to nitrocellulose membrane (Millipore, $0.45 \mu \mathrm{m}$ pore size) at $90 \mathrm{~V}$ for $2 \mathrm{hr}$. The blots were fixed with $25 \%$ isopropanol, $10 \%$ acetic acid for $30 \mathrm{~min}$, and unspecific binding sites were blocked with $10 \%$ nonfat milk powder, $0.05 \%$ Tween 20 for $2 \mathrm{hr}$. The blots were probed with monoclonal antibody Lan 2-3, followed by HRP-conjugated anti-mouse IgG (1:250; Cappel, PA). No immunoreactivity was found in lanes with the void fraction, whereas the binding fraction contained a $130 \mathrm{kDa}$ protein reactive with Lan2-3.

\section{Results}

\section{Anatomical and biochemical overview}

Layoul of the leech serisory afferent systern. The sensory afferents (Fig. $1 A, B$ ) studied in this article are associated with epithelial layers lining the body surface and internal cavities of the leech, 


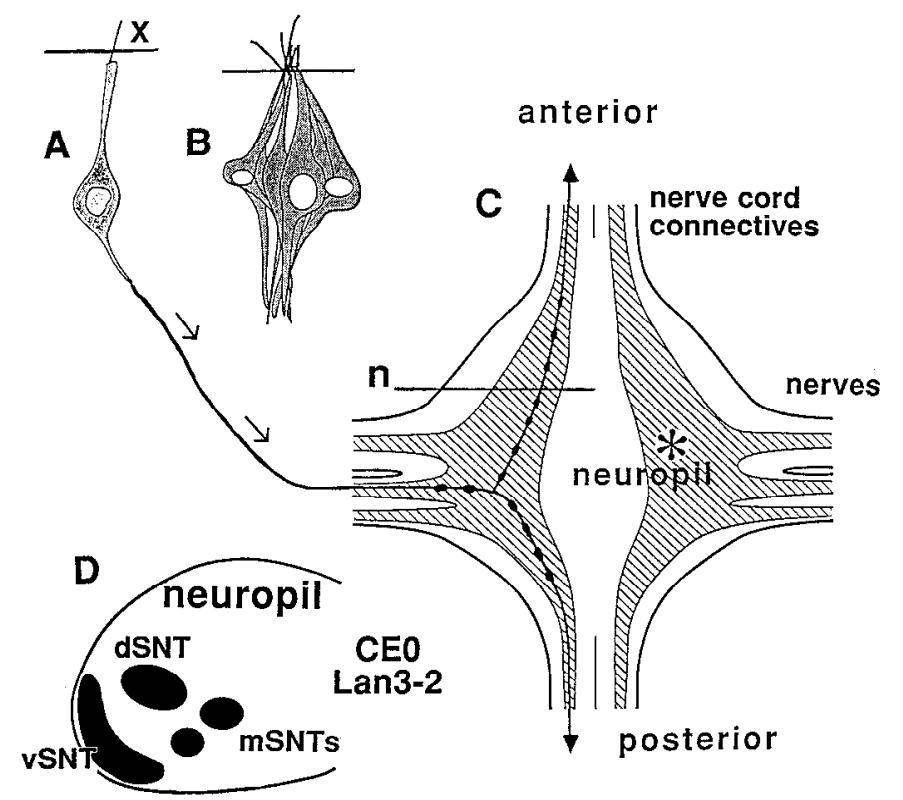

Figure 1. Diagrammatic representation of leech sensory afferent system. The cell bodies of bipolar sensory afferents occur beneath the epithelial layer of the skin or gut, either singly $(A)$ or clustered into sensilla $(B)$. Within a sensory afferent, an environmental signal is transduced via a specialization (e.g., cilia) of its apical process $(x)$, and this afferent signal projects directly to the synaptic areas of the CNS $(C)$ (synaptic ncuropil) via a finc axon (arrows). Within the synaptic ncuropil (asterisk), afferents bifurcate, projecting anteriorly and posteriorly, and display en passant synapses (Fernandez, 1978). Exiting the anterior and posterior poles of the neuropil, sensory afferents project to neighboring ganglia through the connectives that link the segmental ganglia of the nerve cord (CNS) along the length of the body. $D$, Diagrammatic representation of a cross section taken at ganglionic site $n$ illustrates the four sensory neuropil tracts (SNT) into which sensory afferents group their processes, as they are visualized through staining for carbohydrate epitope CEO with Lan3-2 (Peinado et al., 1990): a ventral sensory neuropil tract $(v S N T)$, a dorsal sensory neuropil tract $(d S N T)$ and a pair of medial sensory neuropil tracts ( $m S N T s)$.

and serve as mechano-, chemo-, and heat detectors (Brodfuehrer and Friesen, 1984; Elliott, 1987; C. M. Lent, personal communication). Sensory afferents project to the synaptic areas of the CNS, which consists of a chain of ganglia, via fine axons (arrows, Fig. $1 C$ ). In each body segment, whole populations of sensory afferents densely innervate the synaptic neuropil of a ganglion (asterisk) (Havet, 1899; Sanchez, 1908). In addition, some sensory afferents project into anterior and posterior connectives to innervate ganglia of neighboring segments. As seen in the diagrammatic cross section of the neuropil (Fig. $1 D$ ) taken at plane "n" (Fig. 1C), sensory afferents occupy four discrete tracts in the synaptic neuropil: the ventral, the dorsal, and the two medial sensory neuropil tracts labeled vSNT, dSNT, and mSNTs. The stereotypic pattern of these four sensory neuropil tracts recurs in the head ganglia and all midbody ganglia.

The set and subsets of sensory afferents are encoded by carbohydrate epitopes located on $130 \mathrm{kDa}$ protein. The full extent of the sensory afferent tracts is visualized through staining with monoclonal antibody Lan3-2 (Peinado et al., 1987, 1990), which recognizes a mannose-containing epitope located on $130 \mathrm{kDa}$ sensory afferent protein (Hogg et al., 1983; McKay et al., 1983). In contrast, the three other epitopes investigated here are not shared by all sensory afferents, but rather serve as markers for different subsets of sensory afferents (Peinado et al., 1987). Pre-
Table 1. System of sensory afferents

\begin{tabular}{lll} 
Set or subset & Epitope & mAb \\
\hline Full set & CE0 & Lan3-2 \\
Large subset & CE1 & Laz2-369 \\
Medium subset & CE2 & Liz7-79 \\
Small subset & CE3 & Lan2-3 \\
\hline
\end{tabular}

viously, we showed that two of these subset epitopes (CE1 and CE2, recognized by monoclonal antibodies Laz2-369 and Laz779 , respectively) are located on $\mathrm{N}$-linked carbohydrate chains of $130 \mathrm{kDa}$ sensory afferent protein (Bajt et al., 1990). Here we biochemically characterize the third subset epitope recognized by Lan2-3 (Zipscr and McKay, 1981; Pcinado ct al., 1990). As shown in Figure 2, the third subset epitope is also located on $130 \mathrm{kDa}$ protein, because the monoclonal antibody Lan $2-3$ binds to a broadly smeared band on a Western blot with an apparent molecular weight of $130 \mathrm{kDa}$ (Fig. $2 A$ ). The third subset epitope contains galactose structures, for the binding of Lan2-3 to homogenized CNS protein on dot blots (Fig. $2 B$ ) or to the surface of sensory afferent axons in confocal sections of connectives (Fig. $2 C$ ) is inhibited by $\beta$-methyl galactose. We now refer to the oligosaccharide structures that serve as labels for the full set and subsets of sensory afferents as sequentially numbered carbohydrate epitopes (CE0, CE1, CE2, and CE3, which are recognized by Lan3-2, Laz2-369, Laz7-79, and Lan2-3, respectively). The four different carbohydrate epitopes are cell surface markers, as was demonstrated by staining live sensory afferent neurons (Hogg et al., 1983). Table 1 summarizes the system of sensory afferents with its subsets, as it is encoded by four types of carbohydrate epitopes.

\section{Peripheral distribution of sensory afferent subsets as identified} by markers CE1, CE2, and CE3

Different subsets of lip sensory afferents are identifiable by CE2 and $C E 3$ but not $C E 1$. In the leech lip, sensory afferents are packed into rows of closely spaced sensilla (Fig. $3 A$, arrows). This high density of sensory afferents, which are implicated both in chemodetection (Elliott, 1987) and heat detection (Lent, personal communication), is consistent with the key role these modalities play in the bloodsucking during feeding behavior of Hirudo medicinalis. A higher-power micrograph (Fig. $3 B$ ) shows the elongated cell body of such an afferent with its thick apical process (asterisk) viewed from the side. The apical processes of lip afferents are tipped by multiple cilia (Elliott, 1987), which are occasionally preserved on these immunocytochemically stained afferents.

In order to determine the distribution of different carbohydrate epitopes among lip sensory afferents, we attempted to label lip sensilla with antibodies that recognize the subset markers $\mathrm{CE} 1, \mathrm{CE} 2$, and $\mathrm{CE} 3$. We used juvenile and embryonic leeches for these experiments, because paucity of connective tissue facilitated antibody penctration of lip whole-mount preparations (Thorey and Zipser, 1993). Lip halves from the same embryo were separately labeled for either CE2 or CE3 (Fig. 3C1,C2). The subset of lip sensory afferents positive for CE2 (Fig. 3Cl) is larger than the subset positive for CE3 (Fig. 3C2) (10 experiments). Six further experiments in which lip sensilla were double labeled for CE2 and CE3 demonstrated that these two different subsel carbohydrate epitopes are not expressed on the 


\section{A Western Blot \\ B Dotblot \\ C Confocal Sections}

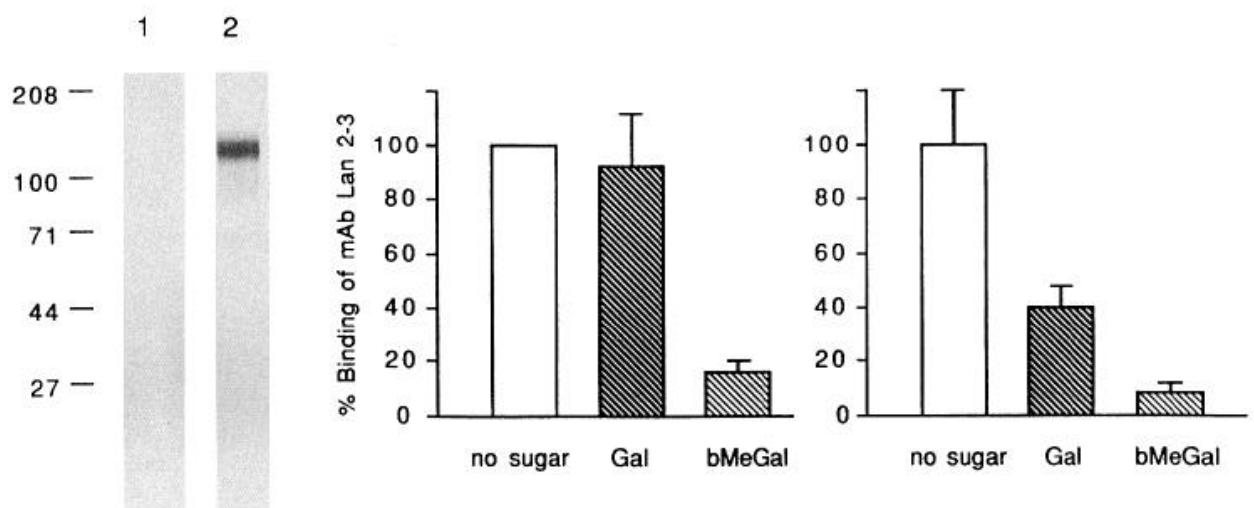

Figure 2. Biochemical characterization of the epitope defining the small subset of sensory afferents. $A$, The low-abundance glycoprotein recognized by monoclonal antibody Lan 2-3 was enriched by lentil lectin affinity chromatography. Leech protein was detergent extracted from 15 nerve cords, chromatographed over lectin agarose columns, and eluted with $\alpha$-methyl mannoside. Following alcohol precipitation, the glycoproteins were solubilized by SDS sample buffer, subjected to SDS-PAGE, and then electroblotted onto nitrocellulose. Probing the blot with Lan2-3 visualized a single broad band centered at $130 \mathrm{kDa}$ (lane 2). Because the fraction prior to the application of $\alpha$-methyl mannoside (lane 1 ) does not bind antibody, the Lan2-3-reactive $130 \mathrm{kDa}$ band in lane 2 is specifically eluted from the lentil lectin column with $\alpha$-methyl mannoside. $B$, The binding specificity of Lan 2-3 was determined by dot blot analysis. Dot blots, prepared in triplicate from nerve cords, were treated with Lan 2-3 in the absence of sugar (no sugar), in the presence of galactose $(\mathrm{Gal})$, and in the presence of $\beta$-methyl galactose ( $\mathrm{bMe} \mathrm{Gal}$ ). Galactose did not inhibit the binding of Lan2-3 to CNS protein. In contrast, $\beta$-methyl galactose inhibited the binding by $80 \%$. $C$, The binding specificity of Lan $2-3$ was determined by analyzing immunocytochemical staining of live sensory afferent axons in the connective. Fifteen serial optical sections were taken from the connective of a nerve cord stained for CE3 afferents with Lan2-3 in the absence and presence of galactose and $\beta$-methyl galactose. The confocal analysis of staining intensity (Odyssey, Noran Instruments) demonstrates the same trend found with dot blot analysis, in that $\beta$-methyl galactose is the strongest inhibitor of Lan2-3 staining.

same neurons (data not shown). In contrast to the above results, we found that the lip sensilla lack sensory afferents that are positive for CE1 (eight experiments). Thus, the lip sensilla are composed of two distinct carbohydrate-coded subsets of sensory afferents.

Gut sensory afferents are identifiable by $C E 1$ but not by $C E 2$ or $C E 3$. The leech gut has sparse sensory innervation. Unlike clustered sensory afferents in the lip and midbody sensilla, sensory afferents in the gut occur in isolation. One probable sensory function of gut afferents is stretch reception, because gut extension leads to cessation of feeding behavior (Lent and Dickinson, 1984). We were able to label sensory afferents in the gut for the subset marker CE1. Figure 4 shows a typical CE1 afferent in the gut. The apical process emanating from its cell body projects toward the gut lumen (Fig. $4 B$ ). Note the bifurcation (arrow) at the distal tip of the apical process as it reaches into the epithelial layer (star) of the gut lumen. Opposite to the apical process, the cell body projects its axon into the muscle layer, where it branches to feed into the rich nerve plexus of CEl axons (Fig. $4 C$ ). No sensory afferents in the gut expressed the subset markers CE2 and CE3 (data not shown; three experiments each). Thus, the gut of leeches is innervated by a subset of sensory afferents that is coded with a carbohydrate epitope distinct from those carbohydrate epitopes found on sensory afferents innervating the lips.

Different subsets of skin sensory afferents are identifiable by $C E 1, C E 2$, and $C E 3$. In the midbody region, skin afferents provide a major sensory input to the synaptic neuropil of the ganglia (Fig. 1). As in the lip, the sensory afferents of the skin are packed into sensilla below the epidermal layer (DeRosa and Friesen, 1981; Phillips and Friesen, 1982). In a hemisegment, the cell bodies of sensory afferents are grouped into seven large and approximately 55 small sensilla (Fig. $5 \mathrm{~A}$ ), which are neatly arranged in both anterior-posterior and lateral-medial dimensions, defined by the ventral and dorsal midline and the five annuli. One known physiological function of sensory afferents in the large midbody sensilla is water wave detection. Using the periodically distributed skin sensilla, the leech is able to orient accurately toward the source of small water disturbances (Friesen, 1981; Brodfuehrer and Friesen, 1984).

We labeled sensory afferent subsets in the skin at late embryonic age (23 d). We found CE1, CE2, and CE3 afferents in every sensilla (Table 2, Fig. $5 B$ ). CE1 afferents formed the largest subset of sensory afferents in both the small sensilla, present on each of the five annuli of a segment, and the large sensilla, unique to the middle annulus. Likewise, CE2 and CE3 afferents form the medium and small subsets, respectively, in both large and small sensilla.

An important question is whether sensory afferent subsets in the skin, as defined by the three different carbohydrate epitopes, are actually disjoint subsets of cells. To address this question, we stained live sensory afferent axons for their different subset carbohydrate epitopes in peripheral nerves and interganglionic connectives of the nerve cord where afferents run as tightly fasciculated bundles (McKay et al., 1983; Peinado et al., 1990). Nerve cords live stained for CE2 and CE3 $(n=3)$ showed the two carbohydrate epitope-defined subsets to be distinct axons in both peripheral nerves and the interganglionic connectives in the 21 midbody segments. CE3 afferents (Fig. 6, lower micrograph) are seen to project as a closely apposed doublet of tracts in the interganglionic connectives. In contrast, in a more dorsal focal plane, CE2 afferents (Fig. 6, upper micrograph) project in tracts that are more widely separated. Thus, these double-labeling experiments demonstrate that CE2 and CE3 

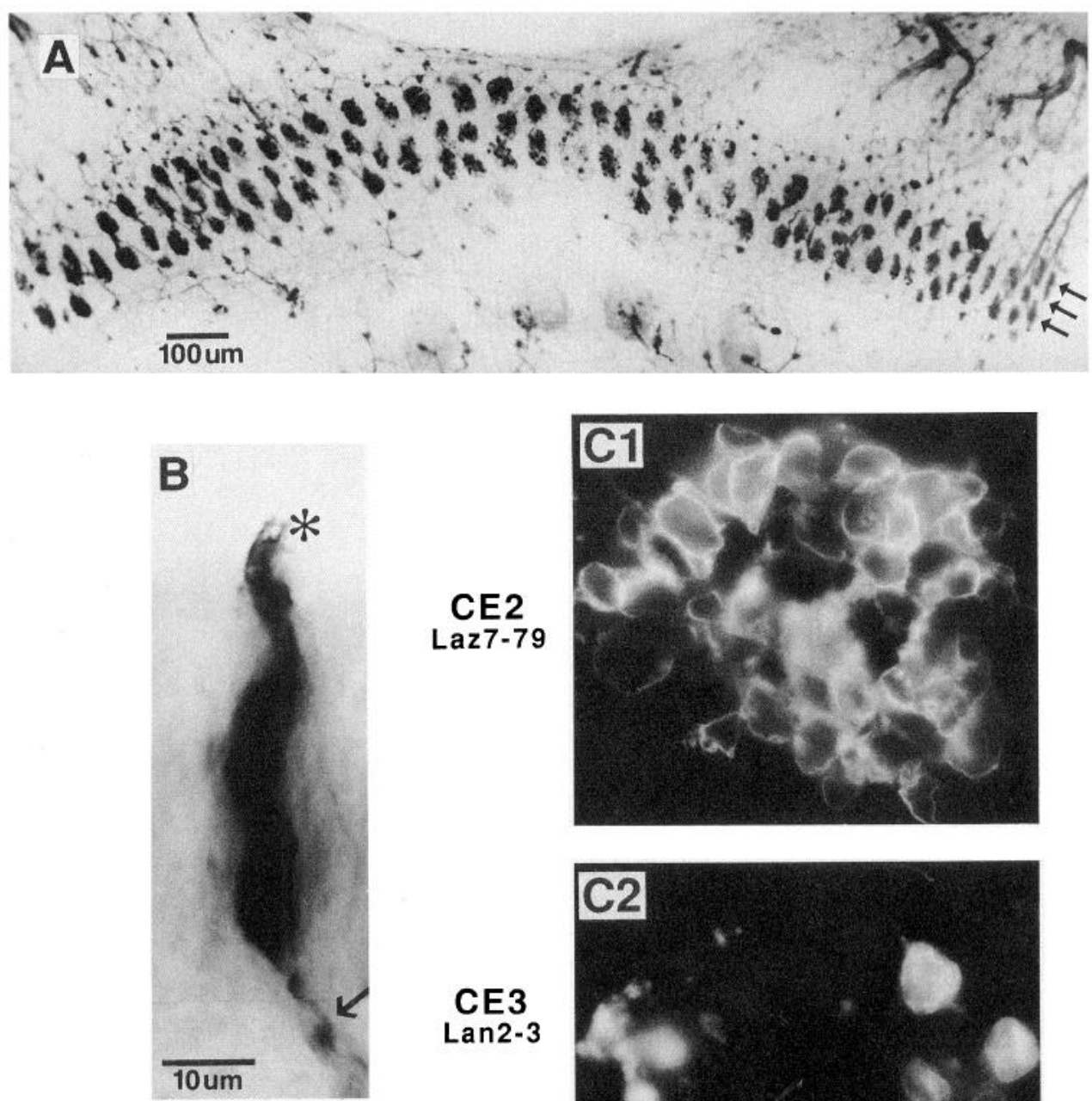

CE2 Laz7-79
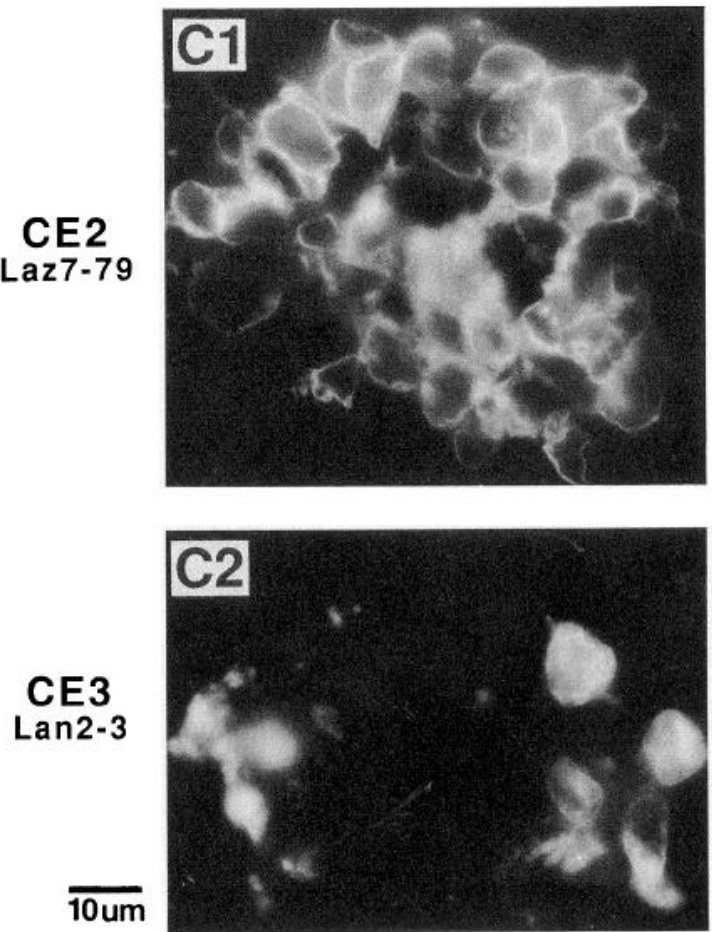

Figure 3. Lip afferents express two subset carbohydrate epitopes, CE2 and CE3. $A$, A low-power micrograph illustrates the distribution of lip afferents into three rows of large sensilla (arrows) in a freshly hatched leech. The afferents are stained here with Laz1-1, which reacts with an internal antigen of lip afferents (Moore et al., 1988). B, The highpower micrograph of the lip of a $12 \mathrm{~d}$ embryo, again stained with Lazl-1, but viewed here from the side, illustrates the bipolar shape of a single lip afferent. The elongated cell body apically projects a process (asterisk) into the environment from which it receives its sensory input and basally projects a single axon (arrow) to carry the sensory input into the CNS. $C l$ and $C 2$, The lip of a $23 \mathrm{~d}$ embryo, which is close to hatching, was stripped of its cuticle and divided into two halves. One half was stained for carbohydrate marker CE2 with Laz7$79(C 1)$ while the other half was stained for carbohydrate marker CE3 with Lan2-3 (C2). The CE2 afferents form a larger subset than the CE3 afferents in the lip. In $A$ and $B$, the primary antibodies were visualized with DAB reaction product using bright-field optics, while in $C l$ and $C 2$ the primary antibodies were visualized with fluorescent labels using epifluorescence. afferents are not only disjoint subsets of neurons in the lips, but continue to belong to disjoint neuronal subsets in the midbody region. In addition, pairwise double labeling nerve cords for CE1 and CE2 $(n=3)$, as well as for CE1 and CE3 $(n=3)$, demonstrated the $\mathrm{CE} 1$ afferents belong to a separate subset (data not shown).

\section{The three carbohydrate-coded subsets contain the majority of the sensory afferents at typical skin sensilla}

The anatomical data collected for this report, particularly those showing carbohydrate epitope-defined afferent subsets to be disjoint subsets, allow us to address the question of whether the three subsets of afferents studied here contain most of the population of epithelium-associated sensory afferents which collectively express CE0, or whether a subset of significant size remains unaccounted. Counting cell bodies of antibody-labeled afferents in the midbody sensilla of $23 \mathrm{~d}$ embryos showed that the sum of the cell bodies expressing the three subset markers $\mathrm{CE} 1, \mathrm{CE} 2$, and CE3 approximately corresponded to the number of cell bodies expressing the full-set carbohydrate epitope, CE0 (Table 2). Note, only a fraction of the carbohydrate epitopelabeled afferents show in the focal plane of this micrograph; counting afferents involved examination of different focal planes. Thus, the sum of the three subsets of afferents approximates the size of the full set.

We also compared the cross-sectional area occupied by the full set and the three subsets of sensory afferent axons in adult leeches. Because the axonal areas were measured in microtome cross sections of the connective, sensory afferents from several different body segments were included. The sum of subset tract areas occupied by CE1, CE2, and CE3 afferents approximately equaled the tract areas occupied by CE0 afferents (Table 2 ).

Thus, the two different measurements of cell bodies and axonal tract area yield the same result: the sum of the carbohydrate epitope-defined subsets corresponds to the size of the full set of afferents. These findings suggest that the three markers CE1, CE2, and CE3 encode all, or nearly all, sensory afferent subsets. Minor sensory afferent subsets, for example, a subset consisting of sparse photoreceptors (Kretz et al., 1976), or afferents from the kidney (Wenning, 1989), may be encoded by still other carbohydrate epitopes. Additionally, a few neurons in each segment are positive for a carbohydrate epitope recognized by monoclonal antibody Lan4-2 (Johansen et al., 1992). Possibly, other carbohydrate epitopes could exist that are shared by in- 
Figure 4. Gut afferents express the subset carbohydrate epitope CE1. A, A camera lucida drawing of a segment of a gut (adult) stained for CE1 with Laz2369 shows the cell body of a single gut afferent and an extensive axonal plexus. The antibody was visualized with DAB reaction product using bright-field optics. Two aspects of this camera lucida drawing are presented in micrographs $B$ and $C$. The round cell body $(B)$ of the sensory afferent projects an apical process whose distal end is bifurcated (arrow). The bifurcation of the apical process reaches into the epithelial layer. The epithelial layer is visualized, because Laz2-369 weakly cross-reacts with epithelial cells (star). From the basal aspect of the cell body, a single axon projects toward the muscle layer where it bifurcates. $C$, The axons in the gut plexus are frequently enlarged to form varicosities (arrows). No sensory afferents in the gut expressed the subset markers CE2 and CE3.

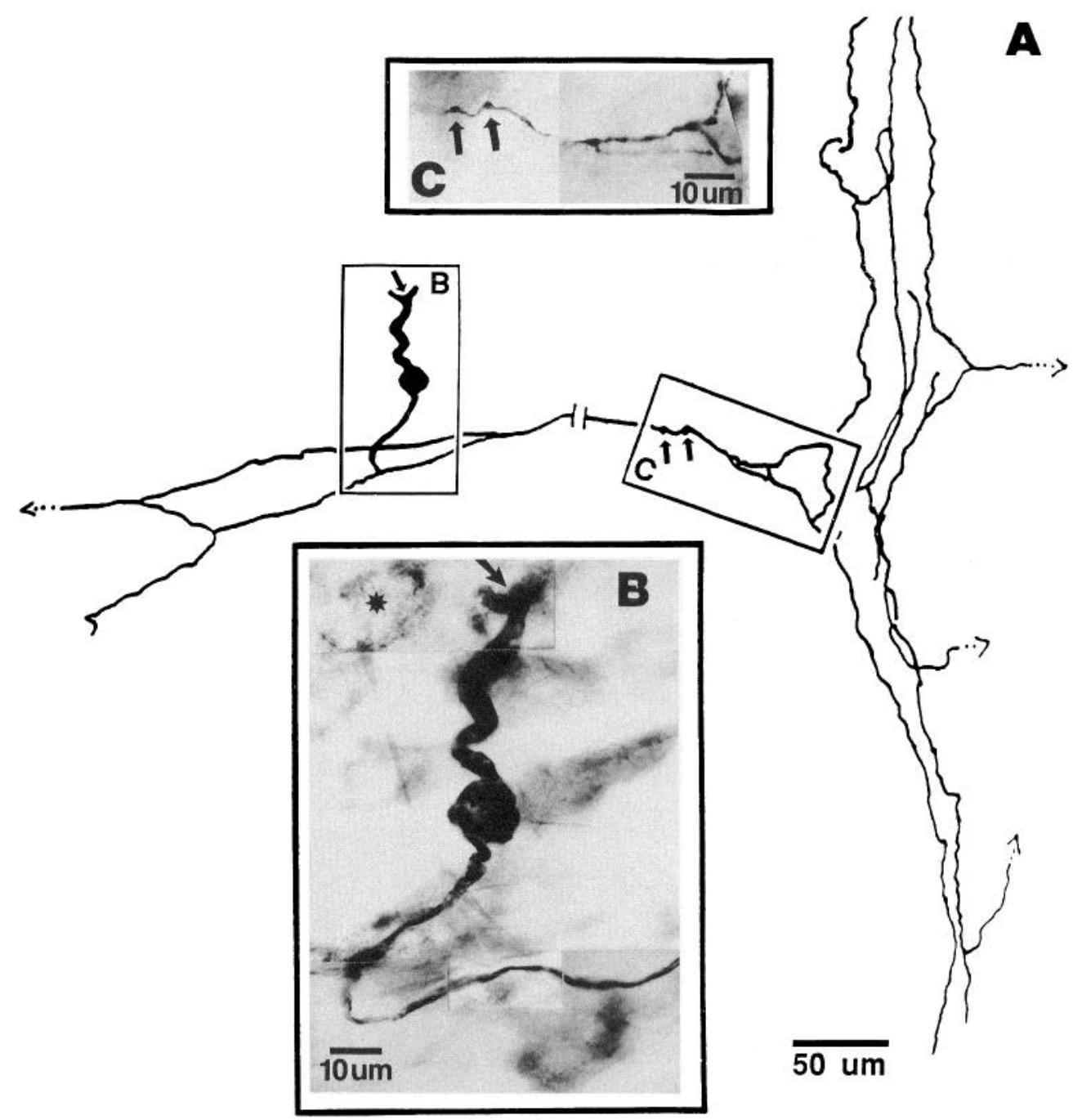

tersecting subsets of these sensory afferents associated with epithelial layers.

\section{CNS distribution of sensory afferent subsets as identified by markers $C E 1, C E 2$, and $C E 3$}

The system of sensory afferent projections to the CNS is as follows. A hemiganglion in a midbody segment receives sensory afferents via peripheral nerves as shown in Figure 7. Here we use the large subset of CE1 afferents as an example to illustrate general features of sensory afferent projection. As the compact bundle of sensory afferents enters the neuropil, it divides into three separate tracts (Fig. 7B2, arrows V, D, M), which then project to different destinations: a ventral, a dorsal, and a pair of medial sensory neuropil tracts (Fig. 7B3, vSNT, dSNT, mSNTs; see also Fig. $1 D$ ). Some afferent axons do not terminate in these sensory neuropil tracts, but project via the anterior (Fig. $7 B 4$ ) or posterior connectives into the ganglia of adjacent segments. Thus, the sensory neuropil of a ganglion is innervated by sensory afferents from multiple segments.

The four tracts occupied by sensory afferents in the synaptic neuropil are referred to as "sensory neuropil tracts" because they differ in their appearance from the tracts of sensory afferent neurons in peripheral and central nerves. Sensory afferents tracking through nerves are fasciculated into tight axonic bun- dles. However, as sensory afferents approach their destinations in the synaptic neuropil, they expand into larger cross-sectional areas, intermingling with the processes of their target neurons. Antibody-stained sensory afferent tracts in the sensory neuropil have a "loose" appearance, because the processes of their postsynaptic neurons are unstained. In his ultrastructural study, Fernandez (1978) noted the large number of synapses formed by sensory afferents with their postsynaptic neurons in these sensory neuropil tracts, which he called fine neuropil. The CE1 afferents occupy most of the ventral sensory neuropil tract but only a fraction of the dorsal and medial sensory neuropil tracts, as seen by comparing the CE1 neuropil pattern (Fig. 7B3) to the CE0 neuropil pattern of the full set of afferents (Fig. 1D), as was previously shown (Peinado et al., 1987).

Comparison of projections of CEI and CE3 afferents in the double-labeled neuropil. Peinado et al. (1990) studied sensory afferents in microtome sections, and reported that the projections of CE1 and CE3 afferents are segregated within the neuropil. Using optical sectioning of whole-mounted ganglia double labeled for subset markers CE1 and CE3, we found that this segregation of the CE1 and CE3 afferents is evident through the entire length of the neuropil. Horizontal optical sections of the ipsilateral neuropil (Fig. 8A1), taken at dorsal, medial, and ventral planes (Fig. $8 A 2$ ), show the projections of the two subsets 

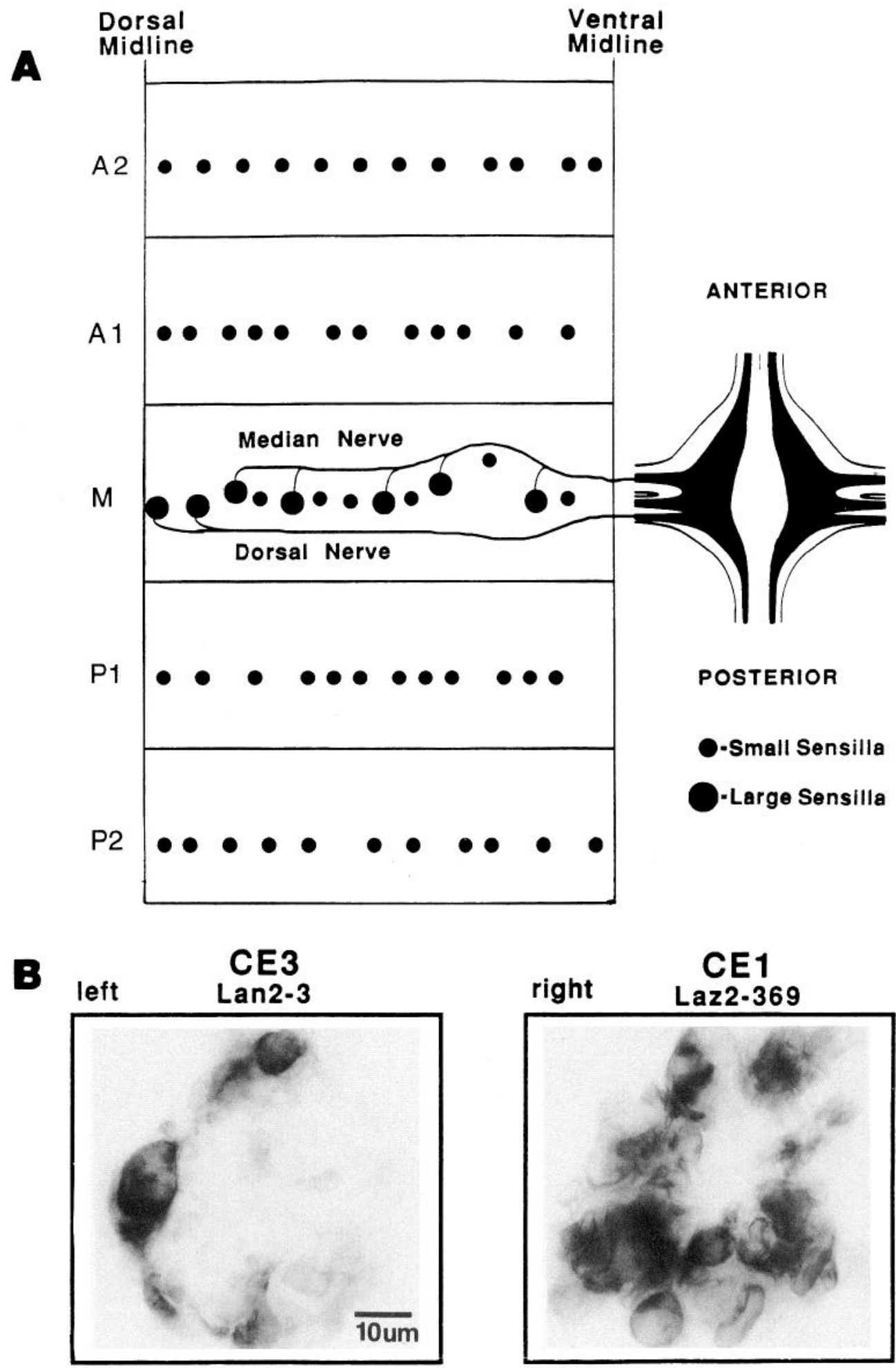

Figure 5. Afferents in midbody sensilla express all three subset carbohydrate epitopes, CE1, CE2, and CE3. A, The body walls of embryos ( $23 \mathrm{~d})$ were stained for CE1, CE2, and CE3 afferents with antibodies Laz2-369, Laz7-79, and Lan2-3, respectively, to determine the distribution of afferents in sensilla of the five annuli comprising a typical hemisegment in the midbody region. The middle annulus $(M)$, which lies adjacent to the ganglion, is seen to contain seven large sensilla and approximately six small sensilla $(6 \pm 1.2 ; n=5$ hemisegments). The afferents in the seven large sensilla project their axons as fasciculated tracts via the median and dorsal nerve into the ganglia. Between 10 and 14 small sensilla $(12 \pm 1.5 ; n=5$ hemisegments) are also found in each of the four annuli $(A 1, A 2, P 1, P 2)$ flanking the middle annulus. The small sensilla project their axons via an extensive nerve plexus (not shown), which eventually connects with the sensory afferent tracts carried in the median and dorsal nerves. $B$, Right and left halves of the body wall of a $23 \mathrm{~d}$ embryo were stained for CE1 and CE3 afferents with antibodies Laz2-369 and Lan2-3, respectively. The primary antibodies were visualized with $\mathrm{DAB}$ reaction product using bright-field optics. The micrographs illustrate cell bodies stained in the third sensillum (S3) of midbody segment 18 . Not all the cell bodies stained in these sensilla are visible in the one focal plane shown. identified by their different subset markers (Fig. $8 B-D$ ). CE1 afferents project heavily into the ventral sensory neuropil tract (vSNT) (Fig. 8B3), as well as into the dorsal and medial sensory neuropil tracts (dSNT and mSNTs). CE3 afferents have heavy projections only to the medial sensory neuropil tracts (mSNTs) (Fig. 8C2).

We noted that the projection patterns of the CE1 and CE3 afferents are not completely segregated. A partial spatial overlap of these carbohydrate-coded subsets occurs in the mSNT, particularly at the level where peripheral nerves enter the neuropil, as seen in the composite drawing (Fig. 8D2). This result was also seen in double-labeled microtome sections (data not shown).

Comparison of projections of CE2 and CE3 afferents in the double-labeled neuropil. In contrast to the mostly segregated projection patterns of CE1 and CE3 afferents, we found that CE2 and CE3 afferents have almost completely convergent pro- 

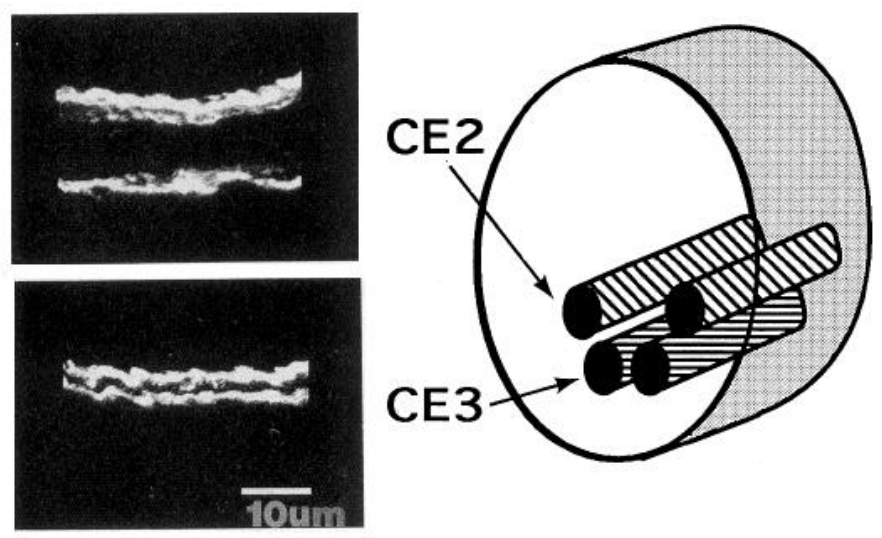

Figure 6. Optical sections illustrating the segregation of CE2 and CE3 axons in the connective. The relative distributions of CE2 and CE3 axons in the connective are illustrated in superimposed optical sections. A live nerve cord from a juvenile leech was double labeled for CE2 and CE3 axons with fluorescein-conjugated Laz7-79 and rhodamine-conjugated Lan2-3, respectively. A connective was horizontally sectioned in $1 \mu \mathrm{m}$ steps going from ventral to dorsal. Eight dorsal serial sections containing the CE2 tracts (upper micrograph) and nine more ventral serial sections containing the CE3 tracts (lower micrograph) were separately summed. The two closely apposed CE3 tracts and two more widely separated CE2 tracts occur in adjacent focal planes as illustrated in the diagram.

jections through the full extent of the neuropil. The relative distributions of projections of the CE2 and CE3 afferents are shown in five superimposed optical sections (Fig. 9) from a ganglion double labeled for the subset markers CE2 and CE3. Both CE2 and CE3 afferents project together into their shared medial sensory neuropil tract (mSNT). However, we found that CE2 afferents (Fig. 9A) also project to the dorsal sensory neuropil tract (dSNT) (asterisk), while the CE3 afferents do not (Fig. 9B).

Comparison of projections of $C E 1$ and CE2 afferents in the double-labeled neuropil. The finding that CE2 afferents project to the dorsal sensory neuropil tract (dSNT) raises the question of how these projections relate to the CE1 afferents, which also project to the same tract. By analyzing double-labeled ganglia with microtome and optical sections, we found that these projections of disjoint subsets often spatially overlap in the dorsal sensory neuropil tract (dSNT), as seen in Figure 10. However, the structure of the dorsal tract is more variable than that of the other synaptic neuropil tracts. Sometimes, the projections of CE1 afferents into the dorsal sensory neuropil tract have a fragmented appearance (Fig. 10), and CE2 afferents may be seen to fill the gaps as shown in the diagram in Figure 11 (data not shown).

\section{Discussion}

Carbohydrate recognition plays an important role in the development of normal projections of leech sensory afferent neurons in the CNS (Zipser et al., 1989; Zipser and Cole, 1991; Song and Zipser, 1993). Carbohydrate epitopes are expressed on the cell surface of sensory afferents, and are located on 130 $\mathrm{kDa}$ membrane proteins. The distribution of the three sensory afferent subsets identified via their different carbohydrate epitopes was examined by labeling of carbohydrate epitopes in peripheral and central nervous tissue, using monoclonal antibodies generated against leech CNS (Zipser and McKay, 1981; Hogg et al., 1983) and partially purified $130 \mathrm{kDa}$ leech protein (Flaster et al., 1983).

Table 2. Comparison of the numbers of cell bodies and axonal area expressing the four different carbohydrate epitopes

\begin{tabular}{|c|c|c|c|}
\hline Set, subsets & $\begin{array}{l}\text { \# of somata, } \\
\text { large sensilla }\end{array}$ & $\begin{array}{l}\text { \# of somata, } \\
\text { small sensilla }\end{array}$ & $\begin{array}{l}\% \text { tract area in } \\
\text { the connective }\end{array}$ \\
\hline $\begin{array}{l}\text { CE0 } \\
\text { Full set } \\
\text { Lan3-2 }\end{array}$ & $120 \pm 11$ & nd & $100 \pm 21$ \\
\hline $\begin{array}{l}\text { CE1 } \\
\text { Large subset } \\
\text { Laz2-369 }\end{array}$ & $53 \pm 7$ & $30 \pm 4$ & $67 \pm 5$ \\
\hline $\begin{array}{l}\text { CE2 } \\
\text { Medium subset } \\
\text { Laz7-79 }\end{array}$ & $41 \pm 4$ & $14 \pm 3$ & $28 \pm 3$ \\
\hline $\begin{array}{l}\text { CE3 } \\
\text { Small subset } \\
\text { Lan2-3 }\end{array}$ & $20 \pm 2$ & $8 \pm 1$ & $19 \pm 5$ \\
\hline $\begin{array}{l}\text { Sum of } \\
\text { subsets }\end{array}$ & $114 \pm 8$ & $53 \pm 5$ & $114 \pm 8$ \\
\hline
\end{tabular}

Embryonic body wall ( $23 \mathrm{~d})$ and adult nerve cords were stained for the full-set carbohydrate marker CE0 with Lan3-2 or for one of the three subset carbohydrate markers CE1, CE2, and CE3 with Laz2-369, Laz7-79, and Lan2-3, respectively. The table lists mean \pm SD values. The cell bodies of antibody-stained sensory afferents were counted in the large sensillum 3 of posterior segments or in neighboring small sensilla. The number of CEO somata in the small sensilla was not determined (nd). The sum of the cell bodies in the large sensilla that bear the three subset carbohydrate epitopes corresponds to the number of cell bodies bearing the CEO epitope, suggesting that the majority of sensory afferents belong to one of the three subsets. We also determined the percentage (\%) tract area in the connective occupied by the sensory afferent axons expressing the four different carbohydrate epitopes. The afferent axons in the connectives originate not only in skin sensilla but also in other organs, for example, the CE1 gut afferents. The area occupied by three subset carbohydrate epitopes approximates the area that is occupied by the axons expressing the full-set epitope CE0, again suggesting that most sensory afferents belong to one of the three subsets. 

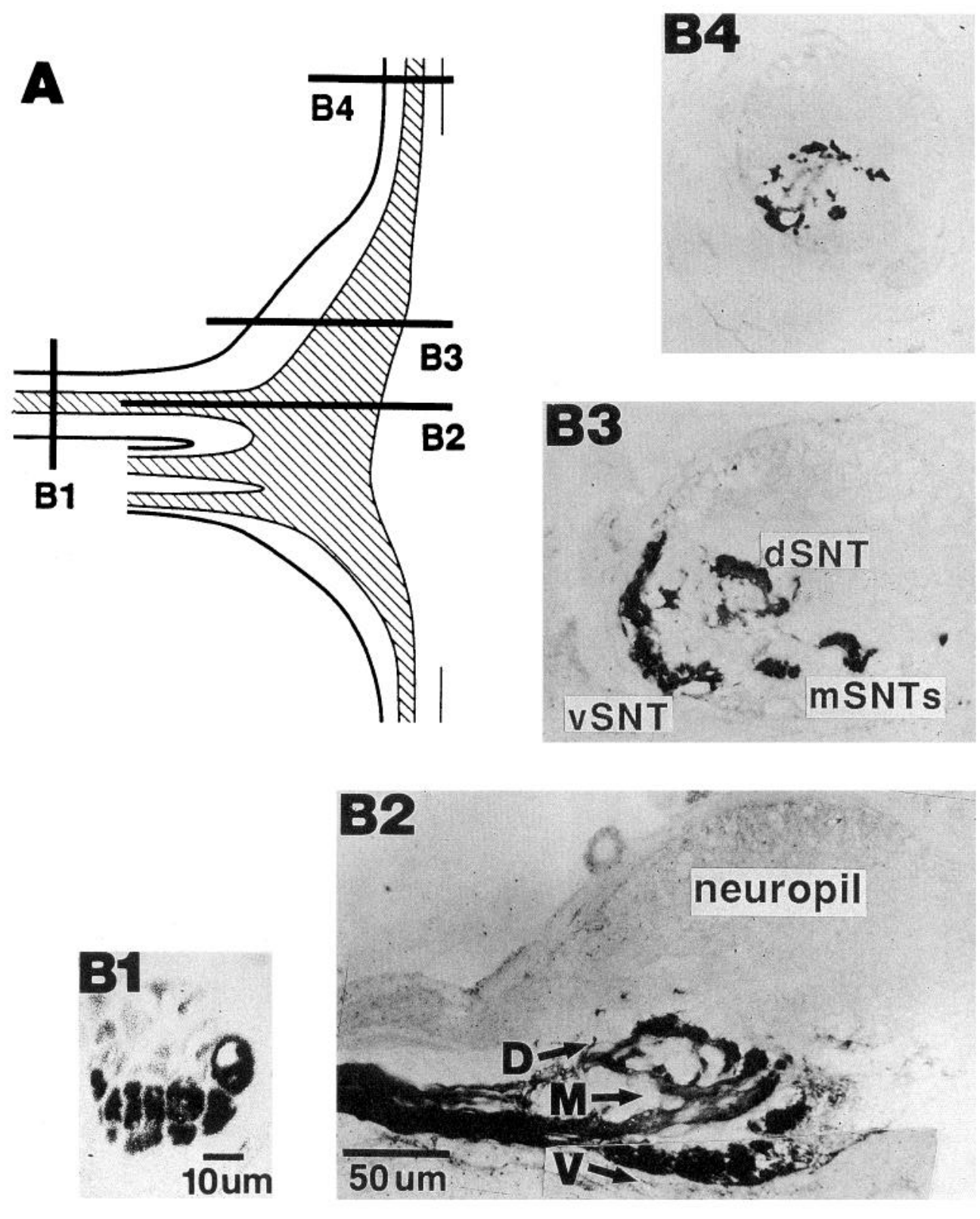

Figure 7. Projections of the large subset of CE1 afferent axons. $A$, The diagrammatic presentation of a hemiganglion indicates at what sites the cross sections of a ganglion stained for CE1 afferents $(B 1-B 4)$ were photographed. Anterior is up. B1 shows sensory afferents projecting through the anterior root nerve as a compact bundle. Individual fascicles of this compact bundle are separated by glial processes (Flaster and Zipser, 1987; Peinado et al., 1990). B2 shows sensory afferents projecting from the root into the neuropil. As the compact CE1 axon bundle exits the root, it divides into three separate tracts-the ventral tract $(V)$, the medial tract $(M)$, and the dorsal tract $(D)$ - which channel sensory afferents to the different sensory neuropil tracts. $B 3$ shows the four sensory neuropil tracts $(v S N T, d S N T$, $m S N T s$ ). $v S N T$ receives afferents via $V$, $d S N T$ receives afferents via $D$, and the two $m S N T$ s receive afferents via $M . B 4$ shows the CE1 axons projecting through the anterior connective. Because sensory afferents project intersegmentally, the sensory afferent axons found in the sensory neuropil tract originate within the periphery of the same segments, as well as in the periphery of other anterior and posterior segments. $B 2, B 3, B 4$ are the same scale.
Double labeling of afferent cell bodies and axons showed that the three subset epitopes are distributed on separate neurons. Labeling of the lip and gut showed a segregation of CE2 and CE3 afferents (found on different cell bodies in the lip sensilla) from CEl afferents (found on cell bodies in the gut) (Fig. 3 vs Fig. 4). Interestingly, although midbody skin sensilla host all three subsets of afferents defined by their carbohydrate epitopes, examination of the projections of these midbody afferents in the CNS neuropil reveals a pattern that mirrors that seen in lip and gut: CE2 and CE3 afferents have highly convergent projection patterns; CE1 projections are mostly segregated from both of these subsets (Fig. 11). Our data (Table 2) comparing the sum of afferents in each subset to the full set of afferents indicate that these subsets constitute all or nearly all of the sensory afferents (defined as the set of neurons that express CE0).

Because carbohydrate epitopes provide information for the development of proper neuronal networks, we can use the data collected in this report on the characteristic distribution of each afferent subset in peripheral tissue and in the CNS neuropil to address the question, what is the nature of the information which carbohydrate epitopes code in this process? We will consider three simple hypotheses to answer this question.

Under the first hypothesis, carbohydrates could code information about the somatopic position of sensory afferents on the body. This hypothesis fits our data poorly. Because sensory afferents expressing the three carbohydrate epitope-defined subsets populate each sensilla in the skin (i.e., both large and small sensilla radially around the body in each segment), the subset carbohydrate epitopes would provide no specific information about the position of these neurons. In the lip, CE2 and CE3 afferents are found in each sensilla, and thus these subset carbohydrate markers could not code position within the lip. Finally, although CE1 is not found in the lip, and CE2 and CE3 are not found in the gut, these markers would not unambigu- 
CE1

Laz2-369

A1

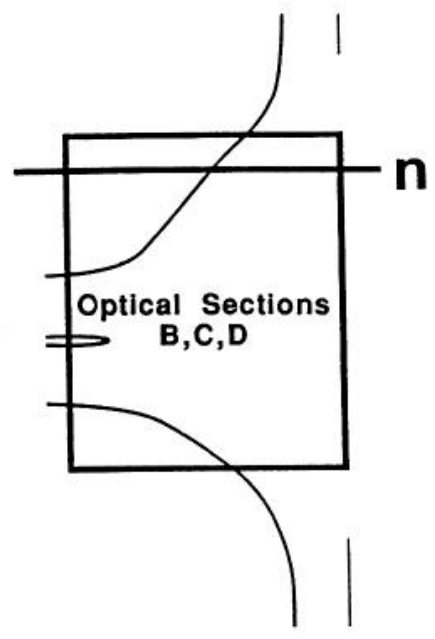

A2

\section{optical section levels at " $n$ "}

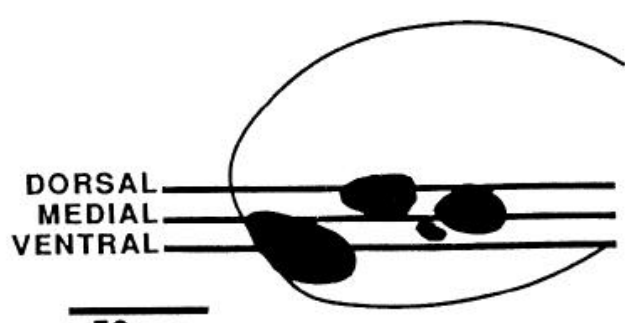

50 um
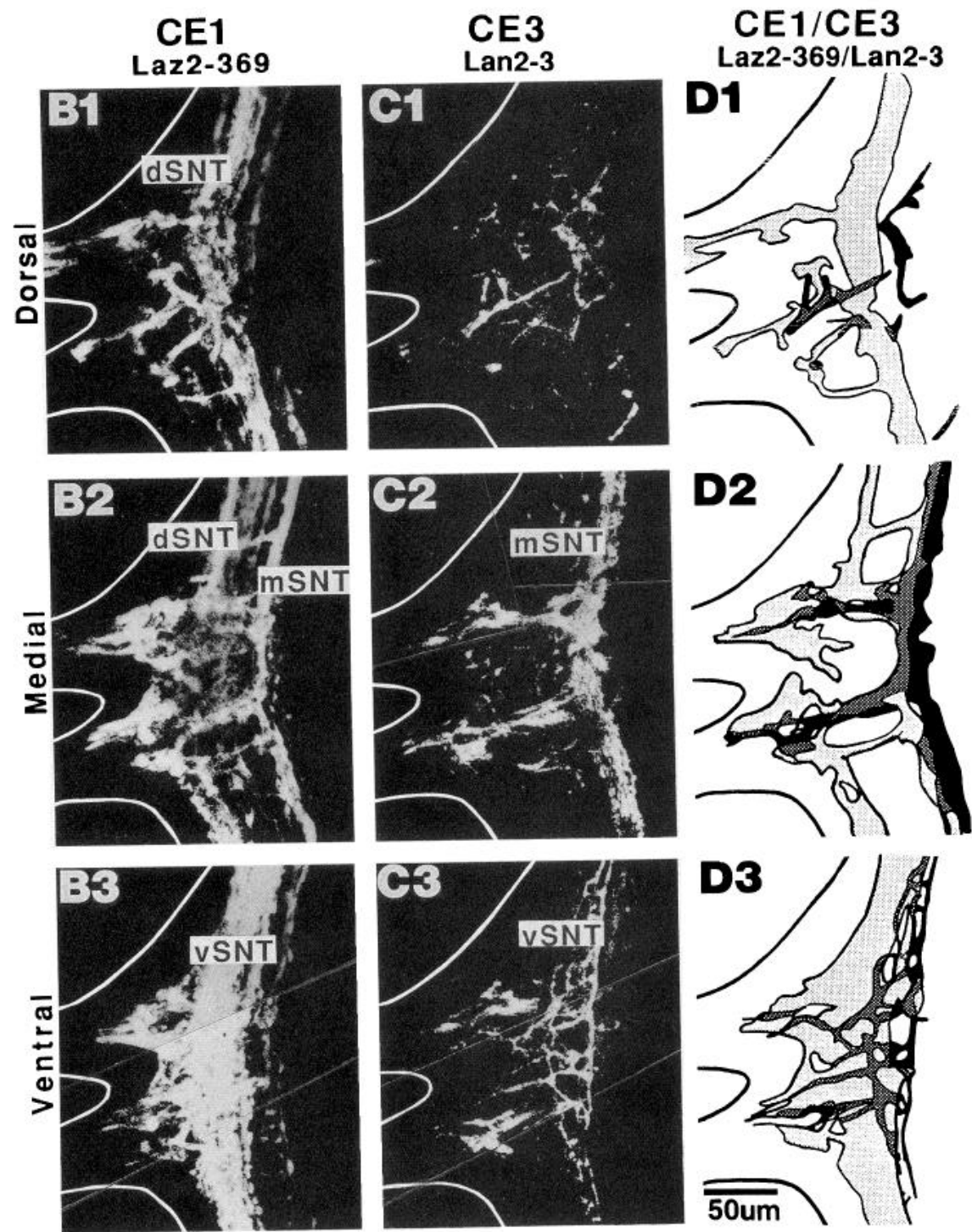

Figure 8. Diagrammatic presentation explaining the locations of the optical sections, and optical sections illustrating the divergence of CE1 and CE3 axons. $A 1$, The diagrammatic presentation of a hemiganglion indicates the extent of ipsilateral neuropil from which the optical sections in Figures $8 B-D$ and 9 were taken. Below $(A 2)$, an additional diagrammatic presentation of a cross section of a neuropil at point $n$ illustrates the three horizontal planes at which optical sections illustrated in Figure $8 B-D$ were taken (Zeiss confocal microscope). The dorsal section contains the dorsal sensory neuropil tract (dSNT), the medial section contains the dorsal and medial sensory neuropil tracts (dSNT and mSNTs), and the ventral section contains the ventral sensory neuropil tract (vSNT). B1-D3, Illustrations show CE1 and CE3 axons in the same ganglion extending from the anterior nerve root to the anterior pole of the ganglion. The adult ganglion was double labeled with Laz2-369 and Lan2-3 to visualize CE1 and CE3 axons, respectively, and optically sectioned for an ipsilateral half of neuropil in three different planes $(B 1-D 3)$. The relative distributions of CE1 and CE3 axons are illustrated in different optical sections in $B(C E 1)$ and $C(C E 3)$. A diagrammatic composite $(D)$ shows the CE1 axons (light gray), CE3 axons (black), and their regions of spatial overlap (dark gray). In the dorsal optical section (BI), CE1 axons project via the dorsal root tract (unlabeled here; see $D$ in Fig. $11 \mathrm{~A}$ ) to the dSNT. In this dorsal plane $(C 1)$ there is only a fine mesh of CE3 axons; as a consequence there is virtually no spatial overlap of the subset's axons at this level $(D 1)$. The medial optical sections contain both the dSNT and mSNT. CE1 axons (B2) are seen in the dSNT and in the mSNT. CE3 axons $(C 2)$ are found only in the mSNT and the medial root tracts leading into the mSNT (unlabeled here; see $M$ in Figs. $7 A, 11 A$ ). As diagrammed in the composite $(D 2)$, there is some spatial overlap of the two different axonal subsets in the medial root tracts. In the mSNT, CE3 axons generally project more medially than the CE1 axons (D2), as had been reported earlier (Peinado et al., 1990). However, in the mSNT, the two subsets of axons are also not completely exclusive, and there is again some spatial overlap, particularly at the zone of root-nerve entry, into the neuropil. The ventral section $(B 3)$ shows the heavy projection of CE1 axons into the vSNT. At this ventral level, there is only a very sparse projection of CE3 axons $(C 3)$; as a consequence, there is very little spatial overlap of the different axonal subsets $(D 3)$.

ously code for gut versus lip tissue, because they are all found in the skin sensilla.

Under a second hypothesis, subset carbohydrate epitopes could code information about the proper target location for sensory afferent projections in the CNS, important information for development and regeneration of proper connectivity. For carbohydrate epitopes to form an efficient code for the target po- sition of different sensory afferent projections, a prerequisite is that subsets of sensory afferents expressing the different carbohydrate epitopes should have spatially distinct distributions. The distribution of sensory afferent projections in the synaptic neuropil is seen in Figure 11, which summarizes our data. As Figure $11 B-D$ shows, the distribution of subset carbohydrate epitopes is redundant (e.g., the disjoint subsets of CE1 and CE2 

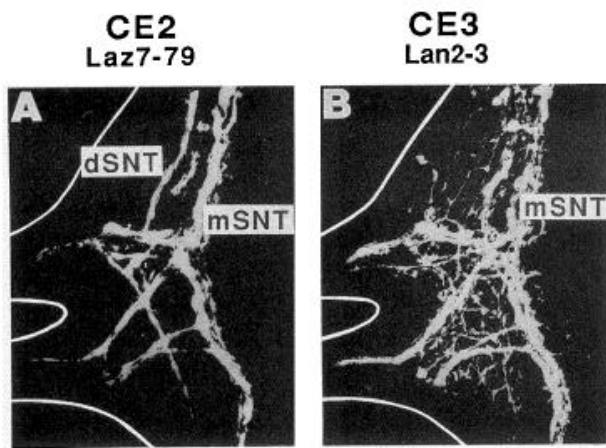

CE2/CE3 Laz7-79/Lan2-3

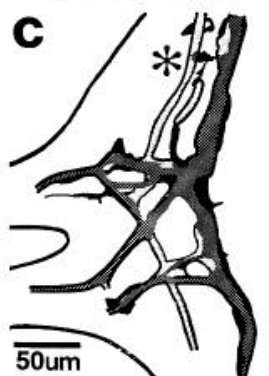

Figure 9. Optical sections illustrating the strong convergence of CE2 and CE3 axons. The relative distributions of CE2 and CE3 axons in the neuropil of an adult leech are illustrated in eight superimposed optical sections cut at medial and dorsal planes and superimposed. A, CE2 axons stained by Laz7-79 are seen in dorsal sensory neuropil tract (dSNT) and they also project via the medial root tracts into the medial sensory neuropil tract $(m S N T)$. B, CE3 axons stained by Lan2-3 project only into mSNT, but not into dSNT. $C$, As shown diagrammatically, the two axonal subsets completely spatially overlap in the mSNT and into the horizontal tracts leading into it. In contrast, the dSNT (asterisk) is exclusively occupied by CE2 axons. Another difference between the two axonal subsets is that CE3 axons show small fascicles and even single afferents that leave the major CE3 tracts, providing a limited innervation to the vSNT.

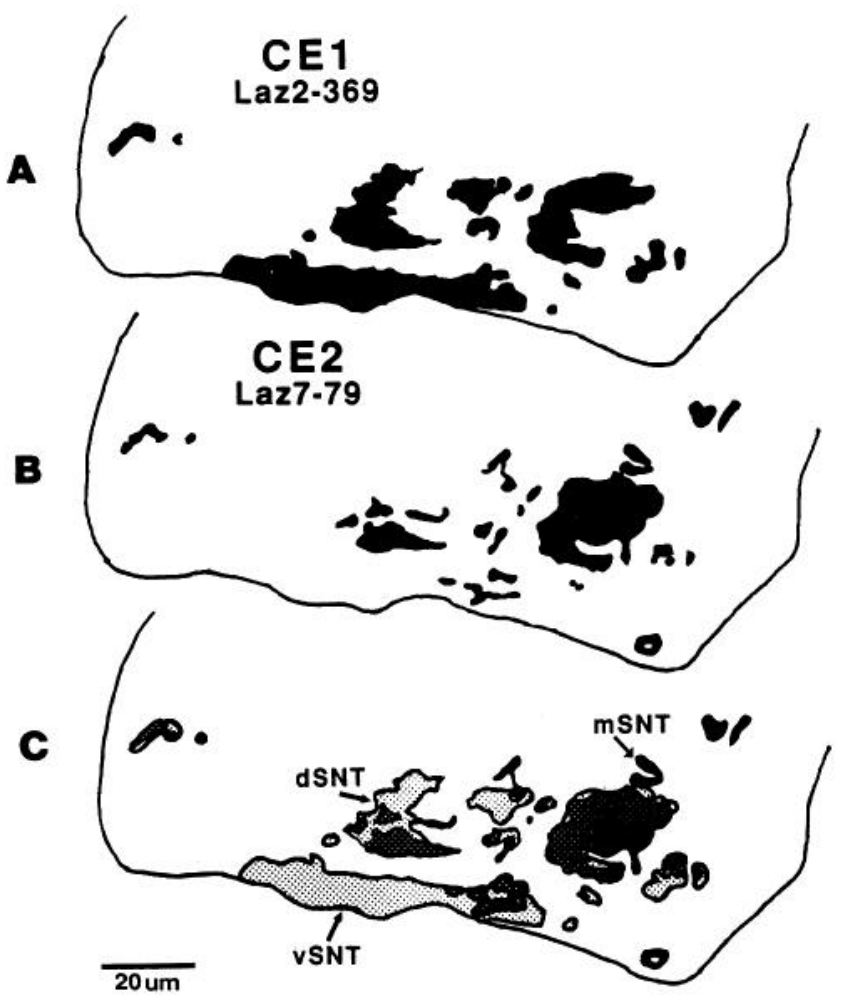

Figure 10. Comparison of sensory afferents positive for CE1 and CE2. The degree to which CE1 and CE2 axons, stained by Laz2-369 and Laz 7-79, respectively, spatially overlap was determined in cryostat sections of double-labeled adult ganglia and by optically sectioning wholemounted ganglia (not shown). The vSNT and some regions of the fragmented dSNT are mostly occupied by CE1 axons $(A)$, which are labeled light gray in the composite $(C)$. The lateral aspect of the mSNT is occupied solely by CE2 axons $(B)$, as indicated in black in the composite (C). The two disjoint axonal subsets spatially overlap in some regions of the dSNT and in the lateral aspect of the mSNT, as indicated in medium gray $(C)$.
A
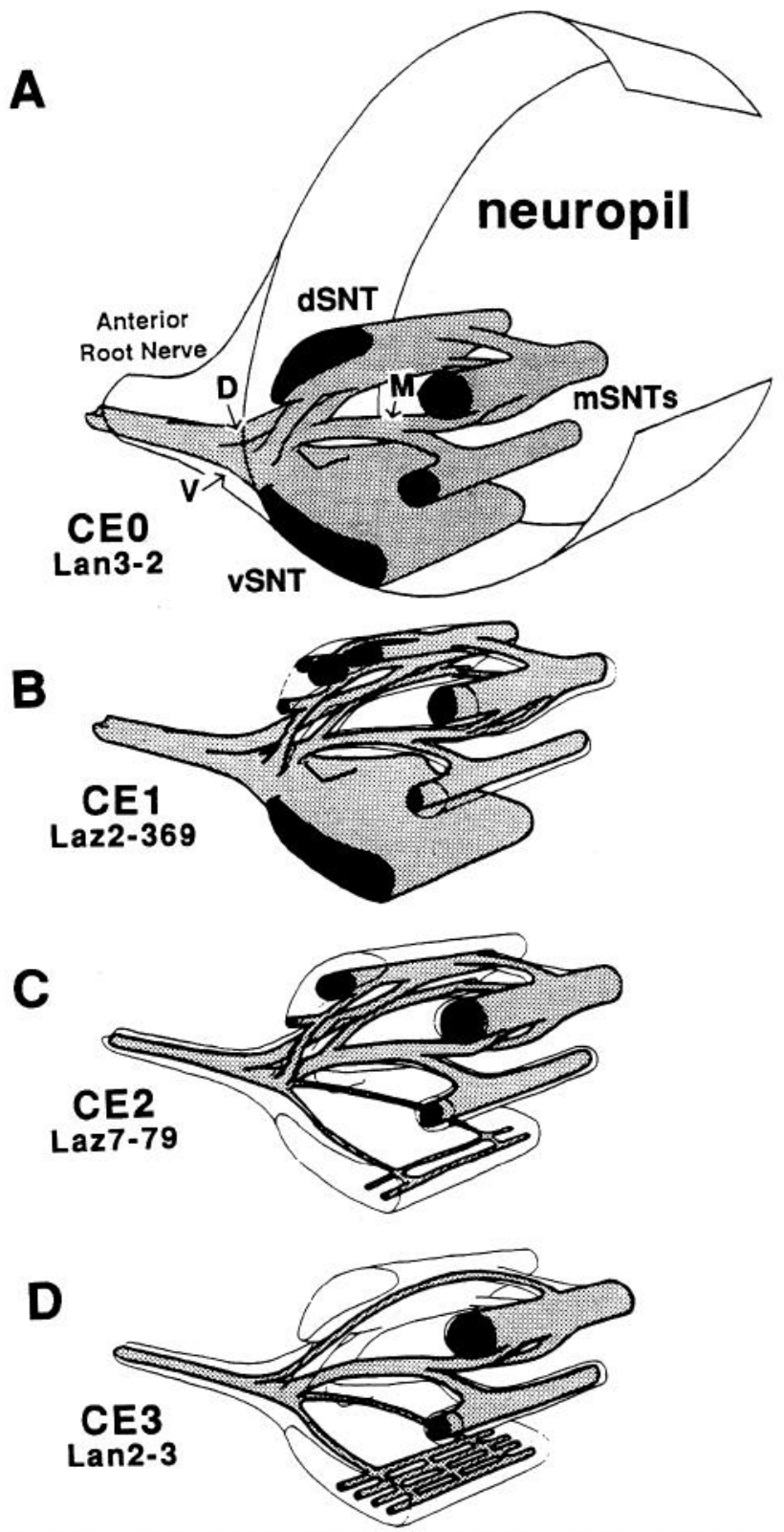

Figure 11. Diagrammatic presentations of the sensory afferents and their subsets in the neuropil. The three-dimensionally drawn diagrammatic presentations are based on extensive data from cross sections (e.g., Figs. 7, 10) and optical sections (e.g., Figs. 8, 9). They present a region of the ipsilateral neuropil starting with the anterior root and extending anteriorly to the anterior border of the ganglion. The neuropil is outlined to indicate that sensory afferents occupy only the anterior, more lateral aspect, of the neuropil. $A$, The full set of sensory afferents, visualized by staining for the full-set carbohydrate epitope CEO with Lan3-2, enters the anterior root nerve as a single tract. The full set projects via the dorsal tract $(D)$ into the dorsal sensory neuropil tract $(d S N T)$, via the ventral tract $(V)$ into the ventral sensory neuropil tract $(v S N T)$, and via the medial tract $(M)$, which bifurcates into the two medial sensory neuropil tracts ( $m S N T S)$. Additionally, there is an unlabeled tract connecting dSNT and the mSNTs. $B-D$, The sensory afferent tracts occupied by CEO axons are outlined. Within these outlines are drawn the axons belonging to large, medium, and small subsets of CE1, CE2, and CE3 axons, labeled by Laz2-369, Laz7-79 and Lan2-3, respectively. Stereotypically, the CE1 axons solidly fill the vSNT, form several interrupted bundles in dSNT, and fill the lateral halves of mSNTs. Stereotypically, CE2 axons occupy most of the mSNTs, the central core of dSNT, and only form a couple of small tracts at the medial border of vSNT. Stereotypically, the CE3 axons occupy most of the mSNTs, form a few tracts at the medial border of the vSNT, and are absent altogether from the dSNT. 
afferents spatially overlap in the dorsal sensory neuropil tract, while the disjoint subsets of.CE2 and CE3 afferents spatially overlap in the medial sensory neuropil tracts). Furthermore, cach subset carbohydrate epitope is found in a variety of different positions within the neuropil (e.g., CE1 afferents have projections to all four sensory neuropil tracts). This projection pattern means that carbohydrate epitopes would code the destination location of their host afferents in a highly ambiguous manner.

Under a third hypothesis, carbohydrate epitopes could code sensory modality information intrinsic to sensory afferents, regardless of their source or target location. The dominant sensory role of skin sensilla, based on physiological and ultrastructural studies (DeRosa and Friesen, 1981; Phillips and Friesen, 1982), is water wave detection; the fact that the dominant sensory afferent subset in skin sensilla expresses CE1 suggests a correspondence between mechanodetection function and this subset. The CE1 afferents are the only sensory afferent subset found in the gut, where sensory afferents also presumably serve as mechanodetectors. Together, the vast majority of the lip sensory afferents express the subset carbohydrate markers CE2 and CE3. The major function of the lip is chemo- and heat detection (Elliott, 1987; Lent, personal communication). Thus, a strong correlation is established between the subset carbohydrate markers CE1, CE2, and CE3 and the sensory modalities, a correlation that may be maintained in skin sensilla: $C E 1$ coding mechanodetection and CE2 and CE3 coding chemo- and heat detection. Under this hypothesis, the convergent projections of CF.2 and CF.3 afferent subsets make sense. The presence of both of these subsets in the lip suggests that they have related, although distinct, functions. Their convergence in the CNS suggests that neural networks, for example, the network controlling feeding behavior, could receive input from both subsets and use carbohydrate epitopes to distinguish inputs from either subset.

The sensory modality coding hypothesis, based on results from widely separated parts of the leech body, implies that the subset carbohydrate epitopes have consistent coding significance through the entire sensory afferent system. For example, we propose that because the CE2 and CE3 lip afferents sense chemical and/or heat stimuli, sensory afferents with the same carbohydrate epitopes in midbody skin sensilla should have similar sensory functions. This is a reasonable proposition because the sensory afferent system, from the neuropil tracts of the head ganglia through the neuropil tracts of all midbody and tail ganglia, is linked by tracts in the intersegmental connectives, and thus forms a continuous network. For example, the CE3 afferent tracts of the head ganglia are thus linked with neuropil tracts in the midbody that express the same subset carbohydrate epitope (Zipser and McKay, 1981). Furthermore, sensory afferents tend to project anteriorly and posteriorly for several segments from their point of entry into the CNS (E. R. Macagno, personal communication), which stresses the importance of a consistent coding scheme for subset carbohydrate epitopes.

Further work that directly correlates carbohydrate markers to ultrastructural and physiological characteristics of the sensory afferents will be needed to test further the hypothesis that subset carbohydrate epitopes code the different sensory functions of sensory afferents.

\section{References}

Bajt ML, Cole RN, Zipser B (1990) The specificity of the 130-kDa leech sensory afferent proteins is encoded by their carbohydrate epitopes. J Neurochem 55:2117-2125.
Brodfuehrer PD, Friesen WO (1984) A sensory system initiating swimming activity in the medicinal leech. J Exp Biol 108:341-355.

Cole RN, Morell RJ, Zipser B (1989) Glial processes, identified through their glial-specific $130 \mathrm{kDa}$ surface glycoprotein, are juxtaposed to sites of neurogenesis in the leech germinal plate. Glia 2:446-457.

DeRosa SY, Friesen WO (1981) Morphology of leech sensilla: observations with the scanning electron microscope. Biol Bull 160:383393.

Elliott E (1987) Morphology of chemosensory organs required for feeding in the leech Hirudo medicinalis. J Morphol 192:181.

Fernandez J (1978) Structure of the leech nerve cord: distribution of neurons and organization of fiber pathways. J Comp Neurol 180:165191.

Flaster MS, Zipser B (1987) The macroglial cells of the leech are molecularly heterogeneous. J Neurosci Res 17:176-183.

Flaster MS, Schley C, Zipser B (1983) Generating monoclonal antibodies against excised gel bands to correlate immunocytochemical and biochemical data. Brain Res 277:196-199.

Friesen WO (1981) Physiology of water motion detection in the medicinal leech. J Exp Biol 92:255-275.

Harlow E, Lane D (1988) Antibodies: a laboratory manual. Cold Spring Harbor, NY: Cold Spring Harbor Laboratory.

Havet J (1899) Structure du systeme nerveux des annilides. Cellule 17:65-136.

Hogg N, Flaster M, Zipser B (1983) Cross-reactivities of monoclonal antibodies between select leech neuronal and epithelial tissues. J Neurosci Res 9:445-457.

Jessell TM, Dodd J (1985) Structure and expression of differentiation antigens on functional subclasses of primary sensory neurons. Philos Trans R Soc Lond [Biol] 308:271-281.

Jessell TM, Hynes MA, Dodd J (1990) Carbohydrates and carbohydrate-binding proteins in the nervous system. Annu Rev Neurosci 13:227-255.

Johansen KM, Kopp DM, Jellies J, Johansen IJ (1992) Tract formation and axon fasciculation of molecularly distinct peripheral neuron subpopulations during leech embryogenesis. Neuron 8:559-572.

Key B, Akeson RA (1991) Delineation of olfactory pathways in the frog nervous system by unique glycoconjugates and N-CAM glycoforms. Neuron 6:381-396.

Kretz JR, Stent GS, Kristan WB (1976) Photosensory input pathways in the medicinal leech. J Comp Physiol 106:1-37.

Laemmli UK (1970) Cleavage of structural proteins during the assembly of the head of bacteriophage T4. Nature 227:680-685.

Lent CM, Dickinson MH (1984) Serotonin integrates the feeding behavior of the medicinal leech. J Comp Physiol 154:457-471.

McGlade-McCulloh E, Muller KJ, Zipser B (1990) Expression of surface glycoproteins in leech neural development. J Comp Neurol 299: 123-131.

McKay RDG, Hockfield S, Johansen I, Thompson I, Frederiksen K (1983) Surface molecules identify groups of growing axons. Science 222:678-684.

Moore N, Morell R, Zipser B (1988) Morphology and shared molecular properties of processes in forming peripheral and central axon tracts. Soc Neurosci Abstr 14:872.

Peinado A, Macagno ER, Zipser B (1987) A group of related surface glycoproteins distinguish sets and subsets of sensory afferents in the leech nervous system. Brain Res 410:335-339.

Peinado A, Zipser B, Macagno L (1990) Segregation of afferent projections in the central nervous system of the leech Hirudo medicinalis. J Comp Neurol 301:232-242.

Phillips CW, Friesen WO (1982) Ultrastructure of water-movement sensitive sensilla in the medicinal leech. J Neurobiol 13:473-486.

Sanchez D (1908) El sistema nervioso de los hirudineos. Trab Lab Invest Biol Univ Madrid 7:31-187.

Schwarting GA, Deutsch G, Gattey DM, Crandall JE (1992) Glycoconjugates are stage- and position-specific cell surface molecules in the developing olfactory system. 2. Unique carbohydrate antigens are topographic markers for selective projection patterns of olfactory axons. J Neurobiol 23:130-142.

Scott SA, Patel N, Levine JM (1990) Lectin binding identifies a subpopulation of neurons in chick dorsal root ganglia. J Neurosci 10: 336-345.

Song J, Zipser B (1993) A neuronal subset selects its target domain via a multistep process that involves the appropriate sequence of recognition of specific saccharides. Soc Neurosci Abstr 19:643.

Thorey I, Zipser B (1993) Different forms of $130 \mathrm{kDa}$ connective tissue 
protein are specific for boundaries in the nervous system and basement membrane of muscle cells in leech. J Neurobiol 24:1531-1542.

Wenning A (1989) Properties of a set of internal receptors in the medicinal leech: the nephridial nerve cells monitor external chloride concentration. J Exp Biol 143:115-132.

Whitlock KE (1993) Development of Drosophila wing sensory neurons in mutants with missing or modified cell surface molecules. Development 117:1251-1260.
Zipser B, Cole RN (1991) A mannose-specific recognition mediates the defasciculation of axons in the leech CNS. J Neurosci 11:34713480.

Zipser B, McKay R (1981) Monoclonal antibodies distinguish identifiable neurons in the leech. Nature 289:549-554.

Zipser B, Morell R, Bajt ML (1989) Defasciculation as a neuronal pathfinding strategy: involvement of a specific glycoprotein. Neuron $3: 621-630$. 